\title{
Pro-angiogenic microRNA-296 upregulates vascular endothelial growth factor and downregulates Notch1 following cerebral ischemic injury
}

\author{
JIE FENG ${ }^{1}$, TIANXIANG HUANG ${ }^{2}$, QING HUANG ${ }^{1}$, HUA CHEN $^{1}$, YU LI ${ }^{1}$, WEI HE ${ }^{1}$, \\ GUI-BIN WANG ${ }^{1}$, LE ZHANG $^{1}$, JIAN XIA ${ }^{1}$, NING ZHANG ${ }^{1}$ and YUNHAI LIU ${ }^{1}$ \\ ${ }^{1}$ Institute of Neurology; ${ }^{2}$ Institute of Neurosurgery, Xiangya Hospital, \\ Central South University, Changsha, Hunan 410008, P.R. China
}

Received June 8, 2014; Accepted June 3, 2015

DOI: $10.3892 / \mathrm{mmr} .2015 .4436$

\begin{abstract}
The present study examined the association between microRNA (miR)-296 and angiogenesis following cerebral ischemic injury, and the underlying mechanisms. A cerebral ischemic model was established in rats via right middle cerebral artery occlusion. The animals were randomly divided into four groups (baseline, 1 day, 3 day and 7 day). Quantitative polymerase chain reaction and western blot analyses were performed to examine the expression levels of miR-296 and hepatocyte growth factor-regulated tyrosine kinase substrate (HGS), respectively. Angiogenesis was assessed by examining microvessel density. The results demonstrated that miR-296 and angiogenesis were significantly upregulated, while HGS was significantly downregulated following ischemic injury. Adenovirus-mediated overexpression of miR-296 markedly enhanced the formation of capillary-like structures in human umbilical vein endothelial cells, parallel with significantly increased expression levels of vascular endothelial growth factor (VEGF) and VEGF receptor 2, and reduced expression levels of DLL4 and Notch1. The results of the present study provided in vivo and in vitro evidence suggesting that miR-296 promotes angiogenesis in the ischemic brain through upregulating VEGF and downregulating Notch1 following cerebral ischemic injury.
\end{abstract}

\section{Introduction}

Ischemic stroke is the dominant subtype of stroke in China, which leads to high rates of morbidity, disability and mortality (1). Immediate restoration or improvement of reduced

Correspondence to: Dr Yunhai Liu, Institute of Neurology, Xiangya Hospital, Central South University, 87 Xiangya Road, Changsha, Hunan 410008, P.R. China

E-mail: yunhail@163.com

Key words: microRNA, angiogenesis, vascular endothelial growth factor, Notch, cerebral ischemia, microRNA-296 regional cerebral blood supply is critical for improving stroke outcomes and post-stroke functional recovery (2). Therefore, angiogenesis has become a focus in the field of ischemic stroke investigation.

MicroRNAs (miRNAs), which are 21 23 nucleotide non-protein-coding RNA molecules, have been identified as negative regulators of gene expression in a post-transcriptional manner (3). Several previous studies have demonstrated that miRNAs are essential determinants of vascular endothelial cell biology and angiogenesis, and contribute to stroke pathogenesis (4-7). A previous study demonstrated that glioma cells and angiogenic growth factors elevate the level of miRNA (miR)-296 in primary human brain microvascular endothelial cells in vitro (8). Angiogenic growth factor-induced expression of miR-296 contributes significantly to angiogenesis by directly targeting hepatocyte growth factor-regulated tyrosine kinase substrate (HGS) mRNA, which leads to reduced levels of HGS and HGS-mediated degradation of vascular endothelial growth factor receptor 2 (VEGFR2) (8). Thus, the present study hypothesized that miR-296 is involved in post-ischemic angiogenesis in cerebral ischemia, and the role of miR-296 in angiogenesis following cerebral ischemia was investigated.

\section{Materials and methods}

Ethical statement. The rats used in the present study were maintained in a climate-controlled vivarium with a $12 \mathrm{~h}$ light-dark cycle with free access to food and water. Each group consisted of six rats and all treatments were performed under anesthesia. Efforts were made to minimize pain and trauma to the animals. All procedures in the present study were approved by the Ethics Committee of Xiangya Hospital of Central South University (Changsha, China) (protocol no. 34721).

Animal model and identification. A total of 24 male Sprague-Dawley (SD) rats (weighing 250-300 g; aged 8-10 weeks) were obtained from the Experimental Animal Center of Central South University (SCXK 2011-0003), and were randomly divided into four groups ( $n=6 /$ group): Baseline group, 1-day group, 3-day group and 7-day group. The middle cerebral artery occlusion (MCAO)-induced 
focal cerebral ischemia mouse model was conducted as previously described (9). Following anesthetization with $4 \%$ chloral hydrate $(4 \mathrm{ml} / \mathrm{kg}$ via intraperitoneal injection; Beijing Chemical Reagent Company, Beijing, China) the right common carotid artery (CCA), the external carotid artery (ECA), and the internal carotid artery were separated through a ventral midline neck incision. The ECA was ligated and cut $4 \mathrm{~mm}$ distal from the ECA-CCA branch. At $\sim 2 \mathrm{~mm}$ distal from the ECA-CCA branch, a small incision was made in the ECA. A 4-0 nylon filament with a silicone resin-coated tip $(0.23 \mathrm{~mm}$ diameter) was inserted through the incision $18-20 \mathrm{~mm}$ into the internal carotid artery, and the wound was closed. The sham-operated (control) animals were anesthetized and underwent surgery without MCAO. The mice were subsequently placed in a post-operative cage, and kept warm and undisturbed for a minimum of $2 \mathrm{~h}$ observation.

Infarct area assessment. Identification of the ischemic area of the brain was performed, according to the 2,3,5-triphenyl-2h-tetrazolium chloride (TTC; Sigma-Aldrich, St. Louis, MO, USA) staining procedure. The rats were sacrificed $24 \mathrm{~h}$ after the onset of MCAO by intraperitoneal injection with $10 \%$ chloral hydrate $(4 \mathrm{ml} / \mathrm{kg})$, followed by decapitation in order to harvest the brains. The rats then received intracardial perfusion with saline (Beijing Dingguo Changsheng Biotechnology Co., Ltd., Beiijng, China) and the brain was removed and frozen for $\sim 5 \mathrm{~min}$ at $-80^{\circ} \mathrm{C}$. The brains were subsequently cut into $2 \mathrm{~mm}$ sections, which were incubated with $2 \%$ TTC at $37^{\circ} \mathrm{C}$ for $20 \mathrm{~min}$ in the dark. Following staining with TTC, the brain sections were fixed in $4 \%$ paraformaldehyde for $24 \mathrm{~h}$.

Reverse transcription-quantitative polymerase chain reaction $(R T-q P C R)$. Total RNA was isolated from the ischemic cortex tissues using TRIzol reagent (Invitrogen Life Technologies, Carlsbad, CA, USA), according the manufacturer's instructions. The total RNA $(2 \mu \mathrm{l})$ from each sample was added to $25 \mu \mathrm{l}$ reaction mixture, and cDNA was synthesized using a First Strand cDNA Synthesis kit (Invitrogen Life Technologies). qPCR was performed to determine the mRNA expression levels using an ABI PRISM 7500 Sequence Detection system (Applied Biosystems Life Technologies, Foster City, CA, USA) using SYBR green PCR Master Mix (Applied Biosystems Life Technologies), according to the manufacturer's instructions. The PCR mix consisted of: $25 \mu \mathrm{l} \mathrm{SYBR}$ green PCR Master Mix, $0.5 \mu 1 \mathrm{cDNA}, 2 \mu 1$ primer pair $(5 \mathrm{pmol} / \mathrm{ml}$ each primer), $22.5 \mu 1 \mathrm{H}_{2} \mathrm{O}$. The following primers were used: miR-296-RT, 5'-GTCGTATCCAGTGCAGGGTCCGAGGTA TTCGCACTGGATACGACACAGGA-3'; miR-296, forward 5'-GAACTAGGGCCCCCCCTCAA-3'; miR-296, reverse 5'-GTGCAGGGTCCGAGGT-3'; U6, forward 5'-CTCGCT TCGGCAGCACA-3'; and U6, reverse 5'-AACGCTTCACGA ATTTGCGT-3' (Switchgear Genomics, Shanghai, China). The PCR cycling conditions were as follows: $95^{\circ} \mathrm{C}$ for $20 \mathrm{sec}$, followed by 40 cycles at $95^{\circ} \mathrm{C}$ for $3 \mathrm{sec}$ and $60^{\circ} \mathrm{C}$ for $30 \mathrm{sec}$.

Western blot analysis. Each section of ischemic cortex tissue was titrated and homogenized in lysis buffer (Beijing Dingguo Changsheng Biotechnology Co., Ltd.), the cells for western blotting were grown for $48 \mathrm{~h}$ and lysed in radioimmunoprecipitation assay buffer (Beijing Dingguo Changsheng Biotechnology Co., Ltd.) containing a protease inhibitor cocktail (Invitrogen Life Technologies). Equal quantities of the extracted proteins were then separated by $10 \%$ sodium dodecyl sulfate-polyacrylamide gel electrophoresis (Beijing Dingguo Changsheng Biotechnology Co., Ltd.), followed by transfer of the resolved proteins onto nitrocellulose membranes (Hunan Honghui Reagent Co., Ltd., Changsha, China). The membranes were blocked for $1 \mathrm{~h}$ at room temperature in 5\% nonfat dry milk in Tris-buffered saline with 1\% Tween-20 (TBST; Wuhan Boster Biological Technology, Ltd., Wuhan, China) and incubated overnight at $4{ }^{\circ} \mathrm{C}$ with the following primary antibodies: Rabbit polyclonal anti-Notch1 (cat. no. ab8925; 1:500), rabbit polyclonal anti-VEGFR2 (cat. no. ab11939; 1:1,000), rabbit polyclonal anti-VEGFA (cat. no. ab51745; 1:1,000) (Abcam, Cambridge, MA, USA), rabbit polyclonal anti-DLL4 (cat. no. 2589; 1:1,000), mouse monoclonal anti-HGS (cat. no. 15087; 1:1,000) (Cell Signaling Technology, Inc., Danvers, MA, USA), and mouse monoclonal anti-GAPDH (cat. no. sc-367714; 1:1,000) (Santa Cruz Biotechnology, Inc., Dallas, TX, USA). Subsequently, following a standard washing cycle with TBST (5 min, 3 times), membranes were incubated with a horseradish peroxidase-conjugated bovine anti-goat (cat. no. sc-2350), anti-rabbit (cat. no. sc-2370) and anti-mouse (cat. no. sc-2371) secondary antibodies $(1: 5,000)$ for $1 \mathrm{~h}$ at room temperature and washed again with TBST (5 min, 3 times). GAPDH was used as a loading control for all experiments. Quantification of the immunoreactivity corresponding to the bands was performed using integrated optical density analysis using Gel pro version 4.0 (Media Cybernetics, Inc., Rockville, MD, USA). All western blot analyses were repeated a minimum of three times.

Immunohistochemistry. The ischemic brain tissues were fixed in $10 \%$ neutralized formalin (Zhongtian Medical Equipment Co., Ltd., Wuhan, China) and embedded in paraffin blocks (Zhongtian Medical Equipment Co., Ltd.). Sections (5 $\mu \mathrm{m})$ were then prepared for immunohistochemical examination. Following deparaffinization and rehydration, antigen retrieval was performed by boiling in $10 \mathrm{mmol} / \mathrm{l}$ citrate buffer (pH 6.0; Zhongtian Medical Equipment Co., Ltd.) for $10 \mathrm{~min}$. Endogenous peroxidase activity was inhibited by soaking for 30 min in $100 \%$ methanol (Hunan Honghui Reagent Co., Ltd.) containing $0.3 \% \mathrm{H}_{2} \mathrm{O}_{2}$, then the sections were blocked with $2 \%$ bovine serum albumin (Wuhan Boster Biological Technology, Ltd.) in phosphate-buffered saline (PBS) for 30 min prior to incubation with the mouse monoclonal CD105 antibody (Thermo Fisher Scientific, Beijing, China) at a dilution of 1:150 overnight at $4^{\circ} \mathrm{C}$. Following washing with PBS, the sections were incubated in primary antibody enhancer (Thermo Fisher Scientific) for $10 \mathrm{~min}$ and horseradish peroxidase polymer (cat. no. 87-8963; Thermo Fisher Scientific) for $15 \mathrm{~min}$ at room temperature. Following washing with PBS, the sections were incubated with diaminobenzidine (Thermo Fisher Scientific) for $3 \mathrm{~min}$ at room temperature. The sections were counterstained with hematoxylin (Beijing Dingguo Changsheng Biotechnology Co., Ltd.) for $30 \mathrm{sec}$, and the staining was evaluated by two individual observers, in a blinded-manner. 


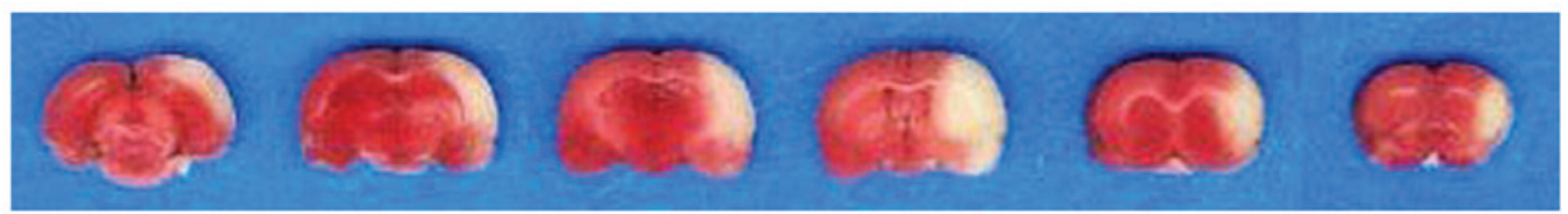

Figure 1. Representative images of rat ischemic brain areas 1 day post-MCAO. Staining of the rat brain tissues using 2,3,5-Triphenyl-2h-tetrazolium chloride was performed at baseline and on days 1, 3 and 7 post-MCAO. The ischemic areas are stained white, while the normal areas are stained red. MCAO, middle cerebral artery occlusion.

Assessment of microvessel density. Quantitative analysis of the microvessel density was performed, as previously described (9). For the determination of microvessel density, the three most vascular areas (hot spots) within a tissue section were selected at a magnification of $\mathrm{x} 40$, and were counted under a light microscope (IX83; Olympus, Beijing, China) at a magnification of $x 100$. The microvessel density was defined as the number of CD105-positive vessels per optical field.

Endothelial cell culture. Human umbilical vein endothelial cell (HUVEC)-12 cells were obtained from the Cell Center of Central South University. The cells were grown in Dulbecco's modified Eagle's medium (DMEM; Gibco-BRL, Gaithersburg, MD, USA), containing 10\% fetal bovine serum (Invitrogen Life Technologies), $100 \mathrm{~mol} / \mathrm{l}$ penicillin and $100 \mathrm{mg} / \mathrm{l}$ streptomycin (Beijing Dingguo Changsheng Biotechnology Co., Ltd.), and incubated at $37^{\circ} \mathrm{C}$ in a humidified atmosphere containing $5 \% \mathrm{CO}_{2}$.

Adenoviral transduction. The HUVEC-12 cells were seeded $\left(2 \times 10^{5}\right.$ cells/well) on six-well plates. On the day of transduction, the cells were harvested and transduced with either the recombinant AdV-Mir-296-green fluorescent protein (GFP) plasmid or with the AdV-GFP plasmid as a control (Promega Corporation, Madison, WI, USA). After 48 h, the cells were harvested and used for subsequent analyses.

$R T-q P C R$. Total RNA was prepared from the HUVEC-12 cells using TRIzol (Invitrogen Life Technologies), according to the manufacturer's instructions. cDNA was synthesized using $15 \mu \mathrm{l}$ total RNA and $2 \mu 1$ oligo (dT) primer in a $25 \mu l$ total reaction volume. RT was performed by incubating the mixture at $42^{\circ} \mathrm{C}$ for $60 \mathrm{~min}$, and the reaction was terminated at $95^{\circ} \mathrm{C}$ for $5 \mathrm{~min}$. The following primers were used: HGS-564, forward 5'-CCA CAATGGCGAGTCTGA-3' and reverse 5'-GAGGGCTGG TAGAGCACA-3'; VEGFA-427, forward 5'-CTACTGCCA TCCAATCGAGA-3' and reverse 5'-CTTTCTCCGCTC TGAGCAA-3'; VEGFR-449, forward 5'-GGGATTGAC TTCAACTGG-3' and reverse 5'-ATGGGATTGGTAAGG ATG-3'; DLL4-362, forward 5'-CAGCAGGGAAGCCAT GAA-3' and reverse 5'-CCGTGGCAATGACACATTCA-3'; Notch1-452, forward 5'-CAAACATCCAGCAGCAGC AA-3' and reverse 5'-AATGCGGGCGATCTGGGACT-3'; and GAPDH-452, forward 5'-ACCACAGTCCATGCCATC AC-3' and reverse 5'-TCCACCACCCTGTTGCTGTA-3' (Invitrogen Life Technologies). The PCR products were then electrophoresed on $1 \%$ agarose gels (Sigma-Aldrich) and stained with ethidium bromide (Beijing Dingguo Changsheng Biotechnology Co., Ltd.).
Tube formation on Matrigel. Matrigel (50 $\mu 1$ of $\sim 10.5 \mathrm{mg} / \mathrm{ml}$; Solarbio Science \& Technology Co., Ltd., Shanghai, China) at $4^{\circ} \mathrm{C}$ was used to coat each well of a 96 -well plate and was allowed to polymerize at $37^{\circ} \mathrm{C}$ for a minimum of $30 \mathrm{~min}$, as described previously (10). The HUVECs cells $\left(5 \times 10^{4}\right)$ were added with $200 \mu 1$ DMEM. Following incubation for $24 \mathrm{~h}$, cultures were visualized (magnification, $\mathrm{x} 400$ ) and images were captured using an inverted microscope (DIAPHOT-TMD; Nikon Corporation, Tokyo, Japan).

Statistical analyses. All statistical analyses were performed using SPSS, version 11.0 (SPSS, Inc., Chicago, IL, USA). Each set of experiments was repeated three times. All continuous variables are expressed as the mean \pm standard deviation. The means between two groups were compared using Student's t-test. Comparisons of means among multiple groups were performed using one-way analyses of variance followed by post-hoc pairwise comparisons using Tukey's test. $\mathrm{P}<0.05$ was considered to indicate a statistically significant difference.

\section{Results}

Establishment of the ischemic injury model. In the present study, cerebral ischemia was induced in rats via MCAO. As shown in Fig. 1, the ischemic areas in the rat brain were stained white, while the normal brain tissues were stained red by TTC staining, which is reported to reflect neurological deficits in the rat brain (11). This indicated that the rat cerebral ischemic injury model had been successfully established.

Angiogenesis increases over time in response to ischemic injury. CD105 is a prominent feature of newly formed blood vessels (12). To assess ischemia-induced angiogenesis in ischemic brain areas, immunohistochemical staining for CD105 was performed in the ischemic rat brain cortex. As shown in Fig. 2, the number of CD105-stained microvessels significantly increased over time following ischemic injury. By contrast, the sham group did not exhibit significant alterations in CD105 staining over time (data not shown). These results indicated that angiogenesis was increased over time in the cortex area following ischemic injury.

Expression of miR-296 increases over time in the ischemic cortex. As shown in Fig. 3, RT-qPCR revealed that the expression levels of miR-296 significantly increased over time in the ischemic brain. This result suggested that miR-296 may be involved in the regulation of post-ischemic angiogenesis. 
A

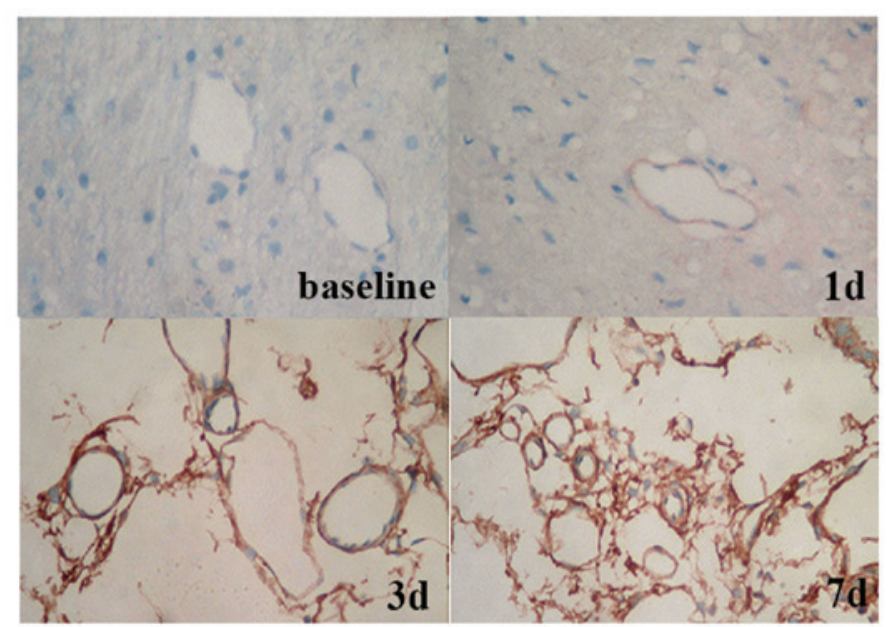

B

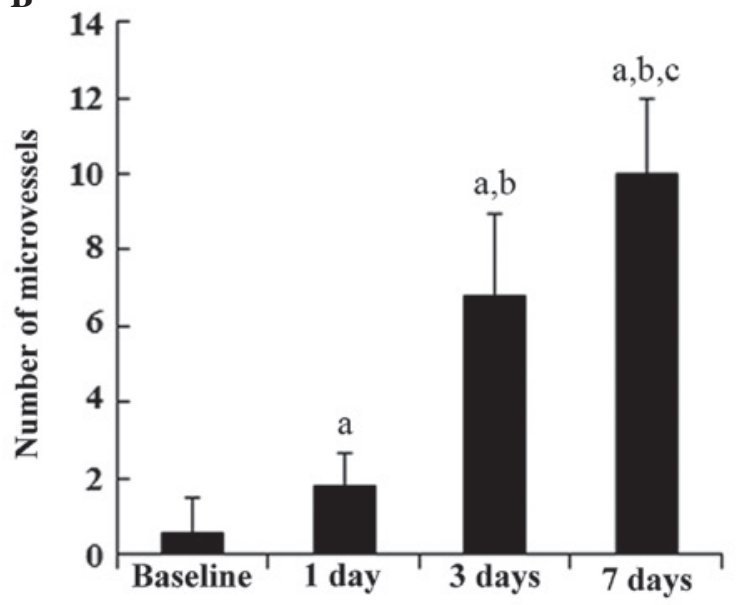

Figure 2. Quantification of CD105-stained microvessels in the rat brain. (A) Representative images of immunohistochemical staining for CD105 in the rat brain (magnification, x400). (B) Quantification of CD105-stained microvessels in the rat brain at baseline and on days 1 (1d), 3 (3d) and 7 (7d) post-ischemic injury. ${ }^{\mathrm{a}} \mathrm{P}<0.05$, vs. baseline; ${ }^{\mathrm{b}} \mathrm{P}<0.05$, vs. 1 day; ${ }^{\mathrm{c}} \mathrm{P}<0.05$ vs. 3 days.

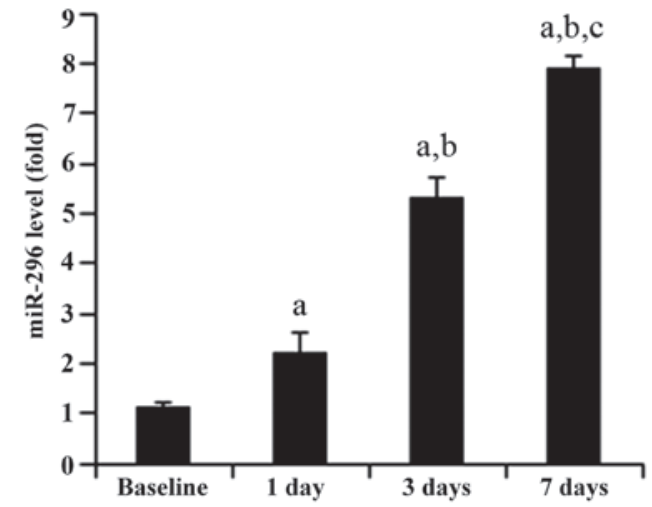

Figure 3. Expression levels of miR-296 in the ischemic cortex. The expression levels of miR-296 were determined by reverse transcription-quantitative polymerase chain reaction at baseline and on days 1,3 and 7 following ischemic injury. ${ }^{a} \mathrm{P}<0.05$, vs. baseline; ${ }^{\mathrm{b}} \mathrm{P}<0.05$, vs. 1 day; ${ }^{\mathrm{C}} \mathrm{P}<0.05$, vs. 3 days. miR, microRNA.
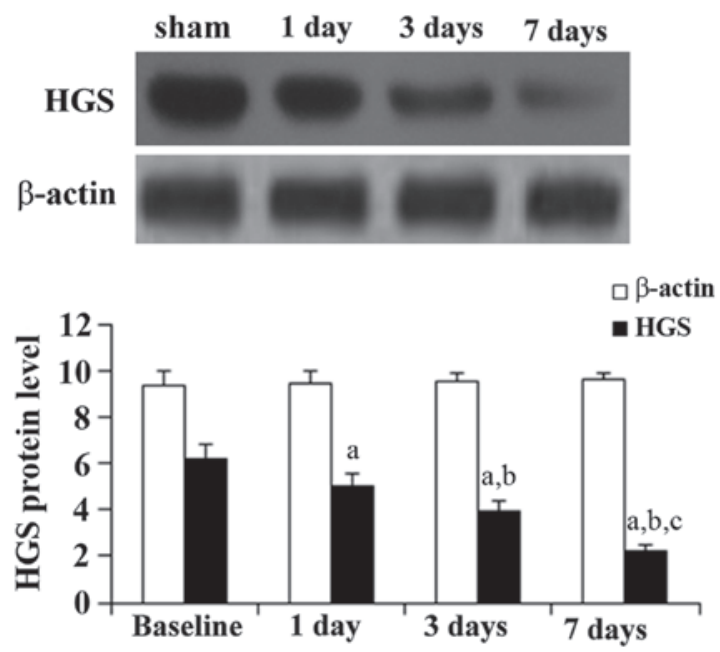

Figure 4. Expression of HGS in the ischemic cortex. Expression levels of HGS were determined by western blot analyses at baseline and on days 1 , 3 and 7 following ischemic injury. ${ }^{\mathrm{a}} \mathrm{P}<0.05$, vs. baseline; ${ }^{\mathrm{b}} \mathrm{P}<0.05$, vs. 1 day; ${ }^{c} \mathrm{P}<0.05$ vs. 3 days. HGS, hepatocyte growth factor-regulated tyrosine kinase substrate.
Expression of HGS reduces over time in the ischemic cortex. To identify miR-296-regulated target genes involved in angiogenesis, the protein levels of HGS following MCAO were measured. As shown in Fig. 4, western blot analyses demonstrated that the expression of HGS reduced over time following the ischemic injury.

Overexpression of miR-296 enhances angiogenesis in vitro. To examine the role of miR-296 in angiogenesis, HUVECs were transduced with adenoviral miR-296 to stably overexpress GFP-tagged miR-296. As shown in Fig. 5, >90\% of the cells expressed GFP compared with the control cells, indicating efficient adenoviral transduction. Tube formation assays demonstrated that the HUVECs stably overexpressing miR-296 (AdV-Mir-296) formed significantly more capillary-like structures $(28 \pm 3.25)$, compared with the untransduced control cells $(12 \pm 2.05 ; \mathrm{P}<0.05$; Fig. 6). These results suggested a role for miR-296 in promoting angiogenesis.

Mechanisms of angiogenesis are mediated by miR-296. To examine the molecular mechanisms underlying miR-296-induced angiogenesis, the expression of the components of the VEGF/VEGFR and DLL4/Notch signaling pathways were examined. As shown in Figs. 7 and 8, compared with the control HUVECs, transduced with the empty adenoviral vector, the mRNA and protein levels of DLL4, Notch1 and HGS were significantly reduced in the HUEVCs stably overexpressing miR-296 $(\mathrm{P}<0.05)$. By contrast, the mRNA and protein levels of VEGF and VEGFR2 were significantly increased $(\mathrm{P}<0.05)$, compared with the control cells.

\section{Discussion}

Ischemic stroke is a leading cause of disability, with few effective treatments available to improve recovery (13). Since degeneration of neurons at the perimeter of the penumbra is reversible, the formation of new blood vessels around the infarcted brain tissue appears to be important in restoring adequate perfusion to the ischemic penumbra (14). 

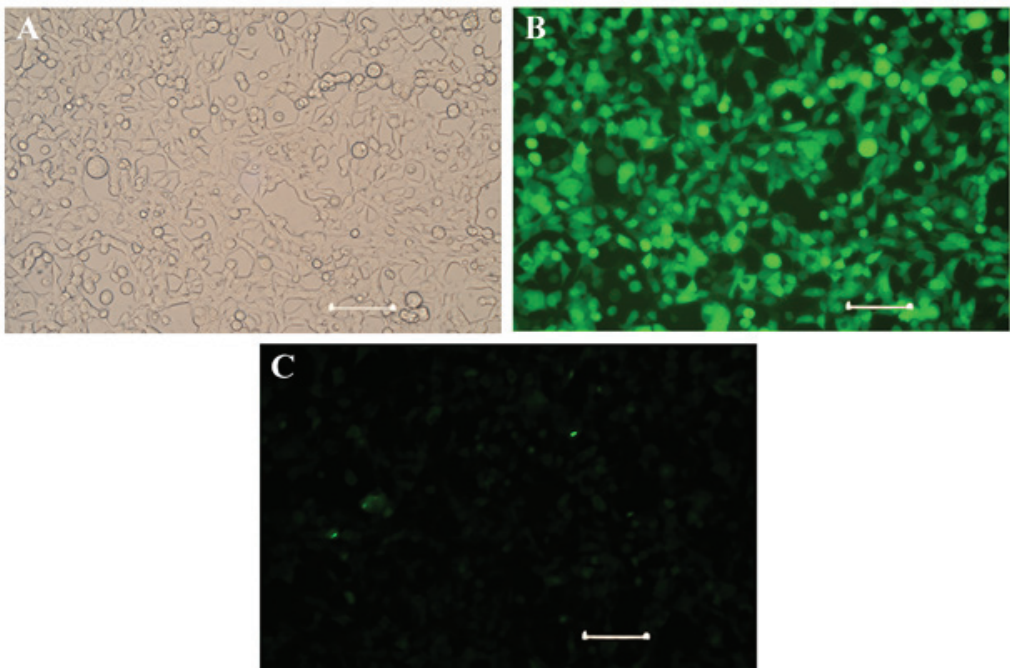

Figure 5. Representative images of GFP staining in human umbilical vein endothelial cells transduced with GFP-tagged microRNA-296. (A) Bright field view; (B) GFP staining; (C) GFP negative control. GFP positivity $>90 \%$ indicated successful construction of the miR-296-expressing adenovirus. Scale bar=20 $\mu \mathrm{m}$. GFP, green fluorescent protein.
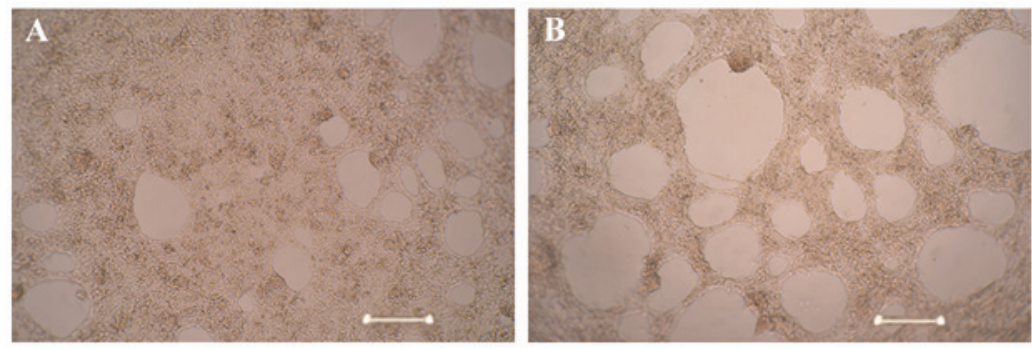

Figure 6. Representative images of capillary-like structures in human umbilical vein endothelial cells stably overexpressing miR-296 (AdV-Mir-296) and untransduced control cells. Overexpression led to apparent tube formation. (A) Untransduced control; (B) endothelial cells stably overexpressing miR-296. Scale bar $=20 \mu \mathrm{m}$. miR, microRNA.

Control miR-296
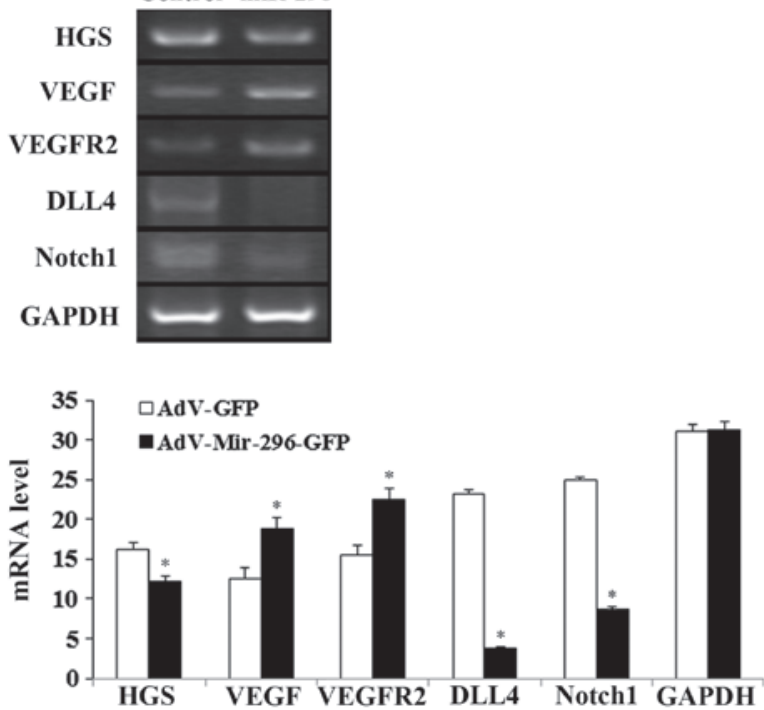

Figure 7. mRNA levels of the VEGF/VEGFR2 and Notch signaling pathways. mRNA levels of VEGF, VEGFR2, Dl14, Notch1 and HGS in human umbilical vein endothelial cells transduced with adenoviral miR-296 (AdV-Mir-296) or empty vector (control/AdV-GFP), determined using reverse transcription-quantitative polymerase chain reaction. GAPDH was an internal control. ${ }^{*} \mathrm{P}<005$, vs. control. VEGF, vascular endothelial growth factor; VEGFR2, VEGF receptor 2; HGS, hepatocyte growth factor-regulated tyrosine kinase substrate; miR, microRNA; GFP, green fluorescent protein.

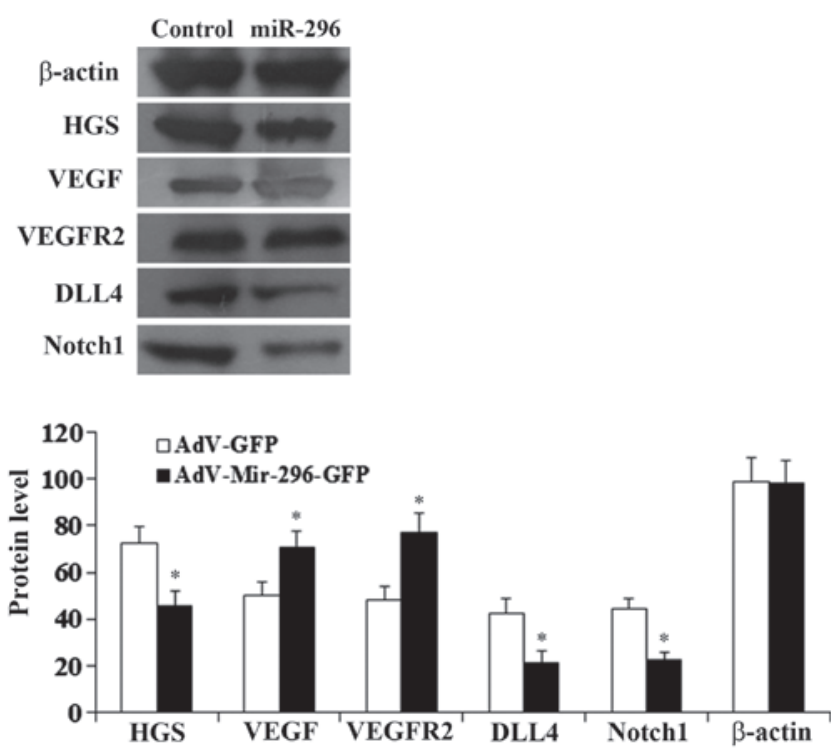

Figure 8. Protein levels of the VEGF/VEGFR2 and Notch signaling pathways. The protein levels of VEGF, VEGFR2, D114, Notch1 and HGS in human umbilical vein endothelial cells transduced with adenoviral miR-296 (AdV-Mir-296) or the empty adenoviral vector (control/AdV-GFP) were determined using western blot analyses. ${ }^{*} \mathrm{P}<005$, vs. control/AdV-GFP. VEGF, vascular endothelial growth factor; VEGFR2, VEGF receptor 2; HGS, hepatocyte growth factor-regulated tyrosine kinase substrate; miR, microRNA; GFP, green fluorescent protein. 
Angiogenesis and vasculogenesis are fundamental processes during formation of new blood vessels, and preclinical and clinical studies have demonstrated that ischemic events in the brain stimulate angiogenesis (15-18). Krupiński et al (19) demonstrated that the number of new vessels in ischemic penumbral regions was correlated with increased survival rates in patients with ischemic stroke, and previous studies have highlighted the importance of angiogenesis in cerebral infarction (20-24).

Due to the variety of mechanisms involved in post-ischemic angiogenesis, a broad understanding of the underlying mechanisms at the cellular and molecular level is important. It has been observed that microRNAs are key in regulating angiogenesis in ischemic diseases (4-7). Endothelial-specific miR-126 has been previously reported to affect the VEGF/VEGFR signaling pathway (5). Although there are several previous reports on miRNAs in cardiac ischemia $(7,25)$, few have focussed on cerebral ischemia (26). A previous study suggested that miR-210 may regulate angiogenesis and the maturation of vasculature in post-ischemic brain tissue (26). In the present study, brain ischemia, induced by MCAO, led to the upregulation of miR-296 in the ischemic cortex, in parallel with a downregulation in the expression of HGS and an upregulation of angiogenesis. These findings are in agreement with a previous study, which indicated that HGS is a target for miR-296 (8). HGS reportedly mediates the degradation of VEGFR2 (27). Thus, miR-296 may increase angiogenesis by promoting VEGF/VEGFR2 signaling through decreasing HGS.

The VEGF and Notch signaling pathways are important in angiogenesis (28-30). In the present study, overexpression of miR-296 was observed to increase the expression levels of VEGF and VEGFR2, and simultaneously reduce the expression levels of DLL4 and Notch1. This was supported by the results of the tube formation assay, which demonstrated that HUVECs stably overexpressing miR-296 formed significantly more capillary-like structure, compared with the untransduced control cells. These results also suggested that there may be another target gene of miR-296, which downregulates the expression of DLL4/Notch. Further investigations are required in order to elucidate the underlying mechanisms.

In conclusion, the present study provided in vivo and in vitro evidence suggesting that miR-296 promoted angiogenesis in the ischemic brain through upregulating VEGF and downregulating Notch1 following cerebral ischemic injury. Thus suggesting that miR-296 may promote angiogenesis following a stroke.

\section{Acknowledgements}

This study was supported by the Fundamental Research Funds for the Central Colleges of Central South University and the Hunan Natural Science Foundation (grant no. 2013zzts094).

\section{References}

1. Wang Y, Liao X, Zhao X, et al: Using recombinant tissue plasminogen activator to treat acute ischemic stroke in China: Analysis of the results from the Chinese National Stroke Registry (CNSR). Stroke 42: 1658-1664, 2011.
2. Jauch EC, Saver JL, Adams HP Jr, et al: Guidelines for the early management of patients with acute ischemic stroke: A guideline for healthcare professionals from the American Heart Association/American Stroke Association. Stroke 44: 870-947, 2013.

3. Zhang C: MicroRNomics: A newly emerging approach for disease biology. Physiol Genomics 33: 139-147, 2008.

4. Zhang T, Weilang X, Liu Y, et al: MicroRNAs and stroke: A brief summary of recent research progress. J Mol Diagn Ther 4: $1-10,2010$.

5. Wang S, Aurora AB, Johnson BA, et al: The endothelial-specific microRNA miR-126 governs vascular integrity and angiogenesis. Dev Cell 15: 261-271, 2008.

6. Fasanaro P, D'Alessandra Y, Di Stefano V, et al: MicroRNA-210 modulates endothelial cell response to hypoxia and inhibits the receptor tyrosine kinase ligand Ephrin-A3. J Biol Chem 283: 15878-15883, 2008.

7. Bonauer A, Carmona G, Iwasaki M, et al: MicroRNA-92a controls angiogenesis and functional recovery of ischemic tissues in mice. Science 324: 1710-1713, 2009.

8. Würdinger T, Tannous BA, Saydam O, et al: miR-296 regulates growth factor receptor overexpression in angiogenic endothelial cells. Cancer Cell 14: 382-393, 2008.

9. Goldmacher GV, Nasser R, Lee DY, et al: Tracking transplanted bone marrow stem cells and their effects in the rat MCAO stroke model. PLoS One 8: e60049, 2013.

10. Lobov IB, Renard RA, Papadopoulos N, et al: Delta-like ligand 4 (Dll4) is induced by VEGF as a negative regulator of angiogenic sprouting. Proc Natl Acad Sci USA 104: 3219-3124, 2007.

11. Bederson JB, Pitts LH, Germano SM, et al: Evaluation of 2, 3 , 5-triphenyltetrazolium chloride as a stain for detection and quantification of experimental cerebral infarction in rats. Stroke 17: 1304-1308, 1986

12. Fonsatti E, Altomonte M, Nicotra MR, et al: Endoglin (CD105): A powerful therapeutic target on tumor-associated angiogenetic blood vessels. Oncogene 22: 6557-6563, 2003.

13. Lloyd-Jones D, Adams RJ, Brown TM, et al; American Heart Association Statistics Committee and Stroke Statistics Subcommittee: Heart disease and stroke statistics - 2010 update: A report from the American Heart Association. Circulation 121: e46-e215, 2010.

14. Scheinowitz M: Therapeutic myocardial angiogenesis: Past, present and future. Mol Cell Biochem 264: 75-83, 2004.

15. Krupiński J, Kałuza J, Kumar P, Kumar S and Wang JM: Role of angiogenesis in patients with cerebral ischemic stroke. Stroke 25: 1794-1798, 1994.

16. Marti HJ, Bernaudin M, Bellail A, et al: Hypoxia-induced vascular endothelial growth factor expression precedes neovascularization after cerebral ischemia. Am J Pathol 156: 965-976, 2000.

17. Hamada Y, Gonda K, Takeda M, et al: In vivo imaging of the molecular distribution of the VEGF receptor during angiogenesis in a mouse model of ischemia. Blood 118: e93-e100, 2011.

18. Hedhli N,DobruckiLW,Kalinowski A, etal: Endothelial-derived neuregulin is an important mediator of ischaemia-induced angiogenesis and arteriogenesis. Cardiovasc Res 93: 516-524, 2012.

19. Krupiński J, Kaluza J, Kumar P, et al: Some remarks on the growth-rate and angiogenesis of microvessels in ischemic stroke. Morphometric and immunocytochemical studies. Patol Pol 44: 203-209, 1993.

20. Sun Y, Jin K, Xie L, et al: VEGF-induced neuroprotection, neurogenesis and angiogenesis after focal cerebral ischemia. J Clin Invest 111: 1843-1851, 2003.

21. Harrigan MR, Ennis SR, Sullivan SE, et al: Effects of intraventricular infusion of vascular endothelial growth factor on cerebral blood flow, edema, and infarct volume. Acta Neurochir (Wien) 145: 49-53, 2003.

22. Navaratna D, Guo S, Arai K and Lo EH: Mechanisms and targets for angiogenic therapy after stroke. Cell Adhes Migr 3: 216-223, 2009.

23. Thurston G, Rudge JS, Ioffe E, et al: Angiopoietin-1 protects the adult vasculature against plasma leakage. Nat Med 6: 460-463, 2000.

24. Gertz K, Priller J, Kronenberg G, et al: Physical activity improves long-term stroke outcome via endothelial nitric oxide synthase-dependent augmentation of neovascularization and cerebral blood flow. Circ Res 99: 1132-1140, 2006. 
25. Ghosh G, Subramanian IV, Adhikari N, et al: Hypoxia-induced microRNA-424 expression in human endothelial cells regulates HIF- $\alpha$ isoforms and promotes angiogenesis. J Clin Invest 120: 4141-4154, 2010.

26. Lou Y-L, Guo F, Liu F, et al: miR-210 activates notch signaling pathway in angiogenesis induced by cerebral ischemia, Mol Cell Biochem 370: 45-51, 2012.

27. Ewan LC, Jopling HM, Jia H, et al: Intrinsic tyrosine kinase activity is required for vascular endothelial growth factor receptor 2 ubiquitination, sorting and degradation in endothelial cells. Traffic 7: 1270-1282, 2006.
28. Carmeliet P, De Smet F, Loges S and Mazzone M: Branching morphogenesis and antiangiogenesis candidates: Tip cells lead the way. Nat Rev Clin Oncol 6: 315-326, 2009.

29. Gerhardt H, Golding M, Fruttiger M, et al: VEGF guides angiogenic sprouting utilizing endothelial tip cell filopodia. J Cell Biol 161: 1163-1177, 2003.

30. Siekmann AF and Lawson ND: Notch signalling limits angiogenic cell behaviour in developing zebrafish arteries. Nature 445: 781-784, 2007. 